\title{
Evaluación del cerámico con estructura tipo perovskita $\mathrm{LaFe}_{0,2} \mathrm{Co}_{0,8} \mathrm{O}_{3}$
}

\author{
Evaluation of the Perovskite $\mathrm{LaFe}_{0.2} \mathrm{Co}_{0.8} \mathrm{O}_{3}$
}

\author{
Wilson Rativa Parada ${ }^{a^{*}}$ \\ Jairo Gómez Cuaspud ${ }^{\mathrm{a}}$ \\ Juan Carda Castellób
}

Recepción: 6 de junio de 2016

Aceptación: 28 de diciembre de 2016

\section{Resumen}

Se sintetizó el cerámico $\mathrm{LaFe}_{0,2} \mathrm{Co}_{0,8} \mathrm{O}_{3}$, usando el método de polimerización con ácido cítrico. Las caracterizaciones de los precursores con espectroscopia infrarroja (FT-IR), ultravioleta visible (UV-vis), y análisis térmicos (TGA-DTA), revelaron la presencia de especies tipo citrato y la consolidación de una estructura estable a partir de $\operatorname{los} 700^{\circ} \mathrm{C}$. Análisis de difracción de rayos X (XRD), energía dispersiva de rayos $\mathrm{X}(\mathrm{EDX})$, microscopia electrónica de barrido (SEM) y espectroscopia Raman, confirmaron la consolidación de un sistema cristalino tipo $\mathrm{LaCoO}_{3}$ de fase simple romboédrica, una superficie homogénea y porosa, y tamaño de partícula nanométrico. La evaluación mediante espectroscopia de impedancias (IS), desde temperatura ambiente hasta $900^{\circ} \mathrm{C}$, mostró un material con alta conductividad eléctrica y comportamiento semiconductor.

Palabras clave: Estructura tipo perovskita, Polimerización con ácido citrico, Óxido semiconductor.

\begin{abstract}
The $\mathrm{LaFe}_{0.2} \mathrm{Co}_{0.8} \mathrm{O}_{3}$ ceramic was synthesized using polymerization with citric acid method. Characterizations of the precursors with Fourier transform infrared spectroscopy (FT-IR), ultraviolet-visible spectroscopy (UV-vis), and thermal analysis (TGA-DTA), revealed the presence of species citrate type, and formation of a stable structure from up to $700^{\circ} \mathrm{C}$. Analysis by means of X-ray diffraction (XRD), energy dispersive $\mathrm{X}$-ray (EDX), scanning electron microscopy (SEM) and Raman spectroscopy, confirmed the consolidation of a crystal system $\mathrm{LaCoO}_{3}$ type, of rhombohedral single phase, with homogeneous and porous surface and nanometer particle size. The evaluation by impedance spectroscopy (IS) from room temperature to $900^{\circ} \mathrm{C}$, showed a material with good electrical conductivity and semiconductor type behavior.
\end{abstract}

Keywords: Perovskite Type Structure, Polymerization with Citric Acide, Semiconductor Type Oxide.

\footnotetext{
${ }^{a}$ Universidad Pedagógica y Tecnológica de Colombia, Avenida Central del Norte 39-115, Tunja-Colombia

*Autor de correspondencia: wilson.rativa@uptc.edu.co

${ }^{\mathrm{b}}$ Universitat Jaume I, Avenida Sos Baynat s/n 12071, Castellón de la Plana-España
} 


\section{Introducción}

Las perovskitas son cerámicos con una estructura tipo $A B X_{3}$, donde generalmente $A$ es ocupado por elementos de tierras raras, $B$ por metales de transición y $X$ por el oxígeno o un halógeno. Una de las principales ventajas de esta clase de materiales consiste en la flexibilidad para la incorporación de diferentes cationes en las posiciones $A$ y $B$ de la estructura, y la posibilidad de combinación de los distintos estados de oxidación. Además, tanto en el sitio $A$ como en el sitio $B$ se puede trabajar más de un elemento químico a la vez, hecho conocido como vacancias o excesos de sustitución, lo que le confiere a los cerámicos variadas y diversas propiedades según su composición [1-3].

La red cúbica es la estructura cristalina ideal de la perovskita, presente en el mineral $\mathrm{CaTiO}_{3}$, que posee una configuración octaédrica $B X_{6}$ donde $A$ se sitúa en las esquinas, $B$ en el centro y el oxígeno en cada una de las caras de la celda unitaria. Pero debido a la modificación y variación de los cationes, la perovskita también puede llegar a presentar redes cristalinas ortorrómbicas, romboédricas, triclínicas, monoclínicas y tetragonales. Una de las formas más utilizadas para determinar la estabilidad de la estructura, es el uso del factor de tolerancia de Goldschmidt, que emplea los valores de radio iónico de los elementos que pueden hacer parte del sistema para conocer si el material será estructural y composicionalmente estable. Comunmente para configuraciones ideales, los valores de tolerancia oscilan entre $0,75 \mathrm{y}$ 1,0 [4].

Dentro de todas las configuraciones de la perovskita, los cerámicos derivados de la estructura simple $\mathrm{LaCoO}_{3}$ sobresalen como un grupo de óxidos con destacadas propiedades para los procesos de catálisis y oxidación de combustibles como metano e hidrógeno, encontrando importantes aplicaciones dentro de la tecnología de generación de energía eléctrica a partir de procesos altamente eficientes, por ejemplo como electrodos en pilas de combustible de óxido sólido. Es así como tras décadas de investigación, han demostrado excelente estabilidad química y estructural a altas temperaturas y atmósferas reductoras, excelente conductividad iónica y electrónica, interesante comportamiento magnético, características morfológicas relevantes, y eficiencias de conversión de hidrocarburos que superan el $60 \%$
[5-7]. Además, la presencia de hierro en la posición $B$ de la estructura de cobaltita de lantano en cantidades entre 10-20\% mol, ha logrado la reducción de los fenómenos de volatilización del catión cobalto, la obtención de áreas activas más grandes, fases cristalinas puras, y tamaños de partícula nanométrico [810]. Aun así, las propiedades de esta configuración se ven limitadas por el implemento de métodos convencionales de síntesis, como la reacción en estado sólido y la co-precipitación, que trabajan con tiempos largos y altas temperaturas, lo que le confiere al material tamaños de partícula demasiado grandes, pobre porosidad, reducida área, y alta tendencia a la sinterización y volatilización de los componentes. Por ello, las investigaciones acerca de las rutas de síntesis de química húmeda, como la polimerización con ácido cítrico, que permitan disminuir las temperaturas y tiempos de tratamiento térmico, y aseguren la obtención de sólidos con un tamaño de partícula nanométrico, excelente control sobre la composición trabajada, alta homogeneidad en la morfología, superficies porosas y de gran área superficial específica, han venido ganado bastante interés en los campos de fabricación y aplicación de los cerámicos [11-14].

De esta forma, este trabajo se centra en la síntesis y caracterización del sistema $\mathrm{LaFe}_{0,2} \mathrm{Co}_{0,8} \mathrm{O}_{3}$, mediante el empleo de un método de quimica húmeda basado en una reacción de polimerización con ácido cítrico y una posterior etapa de autocombustión que permite obtener materiales con propiedades relevantes en función del tamaño de partícula y las características superficiales generadas. Para esto, el óxido se obtuvo en dos etapas de reacción, la primera se fundamentó en la generación de un material metalorgánico derivado de la reacción con ácido cítrico, que se caracterizó mediante espectroscopia infrarroja y ultravioleta (FT-IR y UV-vis), así como análisis térmicos (TGA-DTA), que permitieron determinar la efectividad del método de síntesis y la temperatura ideal de consolidación de la fase cristalina buscada. En una etapa posterior, el precursor fue calcinado hasta la obtención de un conglomerado cerámico que fue estudiado de forma estructural y microscópica utilizando técnicas de difracción de rayos X (XRD), espectroscopia Raman, microscopia electrónica de barrido (SEM) y espectroscopia de impedancias (IS), con el fin de evaluar el efecto de la técnica de síntesis en la obtención de propiedades superficiales, texturales, morfológicas, y eléctricas. 


\section{Metodología}

Para la síntesis del sistema $\mathrm{LaFe}_{0,2} \mathrm{Co}_{0,8} \mathrm{O}_{3}$, se partió de disoluciones en concentración $1,00 \mathrm{M}$ de los nitratos de $\mathrm{La}\left(\mathrm{NO}_{3}\right)_{3} .6 \mathrm{H}_{2} \mathrm{O}, \mathrm{Fe}\left(\mathrm{NO}_{3}\right)_{3} .9 \mathrm{H}_{2} \mathrm{O}$, y $\mathrm{Co}\left(\mathrm{NO}_{3}\right)_{3} .9 \mathrm{H}_{2} \mathrm{O}(99,99 \%)$, los cuales se dosificaron en un reactor de vidrio según la composición buscada como ha sido establecido de forma preliminar [15-17]. El sistema acuoso, fue tratado térmicamente a $80^{\circ} \mathrm{C}$ bajo condiciones de agitación magnética (150 rpm) y reflujo por 120 minutos; posteriormente, se adicionó al sistema una disolución de ácido cítrico 2,00 M con una proporción 0,5:1 en relación al contenido total de cationes metálicos, para favorecer la formación de compuestos de coordinación tipo citrato, dando continuidad al reflujo por 60 minutos más para asegurar la formación de los respectivos complejos de coordinación entre los cationes componentes y el ligando citrato. La disolución se trató térmicamente entre 100 y $250{ }^{\circ} \mathrm{C}$ durante seis horas, consiguiendo la evaporación del solvente y la consolidación de un gel denso, que fue tratado a $350{ }^{\circ} \mathrm{C}$ hasta el inicio de un proceso de autocombustión, generando un precursor carbonoso de apariencia heterogénea. Dicho precursor se caracterizó mediante espectroscopia FT-IR y UVvis en un equipo Perkin-Elmer 1600 y en un equipo Spectra 1000, respectivamente. Los análisis térmicos (TGA-DTA), realizados en un equipo Mettler Toledo modelo TGA/SDTA851e/LF/1600, complementaron la información necesaria sobre la temperatura ideal de consolidación de la fase cristalina buscada.

En base a los resultados de las pruebas TGADTA, se realizó un tratamiento térmico del precursor a $850^{\circ} \mathrm{C}$ durante 12 horas bajo condiciones de flujo de aire $\left(10 \mathrm{~mL} \mathrm{mín}^{-1}\right)$, con el fin de eliminar los residuos remanentes del proceso de autocombustión. El polvo cerámico obtenido se molturó en mortero de ágata y se tamizó a malla 400 U.S. estándar. La caracterización estructural se realizó mediante difracción de rayos X, en un equipo Panalytical X'pert PROMPD, con configuración Bragg-Brentano, radiación de $\mathrm{Cu} \mathrm{K} \alpha(\lambda=1,54186 \AA)$, entre 10 y $80^{\circ}(2 \theta)$, y con pasos de $0,02^{\circ}$. La identificación de la fase y la morfología del sólido se confirmaron haciendo la búsqueda en el programa X'Pert High Score y en las bases de datos del International Centre of Diffraction Data (ICDD), mientras que el tamaño de cristal se calculó utilizando la ecuación de Debye-Scherrer ajustada con una constante de 0,89 como referencia, utilizando las señales más intensas. La caracterización por espectroscopia Raman, se hizo en un equipo HR-UV 800 Infinity Microprobe (Jobin-Yvon), equipado con un detector $\mathrm{CCD}\left(-70^{\circ} \mathrm{C}\right)$ y una fuente láser de $10,7 \mathrm{~mW}$, entre 100 y $1100 \mathrm{~cm}^{-1}$.

La caracterización superficial del sólido se realizó mediante microscopia electrónica de barrido (SEM), en un equipo Leica-Zeiss LEO 440 con cañón de electrones y un voltaje de aceleración de 20 $\mathrm{kV}$. La composición química y elemental de los materiales se confirmó mediante análisis por microsonda de energía dispersiva de rayos X (EDX), en un espectrómetro S4 Pioneer Bruker secuencial de rayos $\mathrm{X}$ por dispersión de longitudes de onda. La caracterización eléctrica se efectuó con el uso de espectroscopia de impedancias en un equipo Agilent 4294A bajo condiciones de corriente continua desde temperatura ambiente hasta $900^{\circ} \mathrm{C}$ entre $40 \mathrm{~Hz}$ y $13 \mathrm{MHz}$, para lo cual se prensó isostáticamente $0,05 \mathrm{~g}$ del material a una presión de $7 \mathrm{MPa}$ durante 120 segundos. La pastilla resultante se sinterizó a $900^{\circ} \mathrm{C}$ por 12 horas, y se pulió con lija de carburo de silicio 1200 para mejorar el área de contacto en los electrodos del sistema de medida, después se sometió al procedimiento de eletrodación con platino.

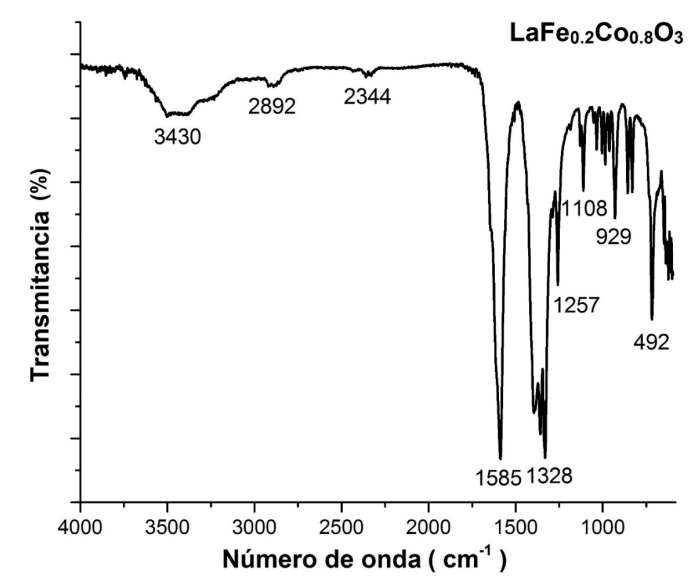

Figura 1. Espectro FT-IR del precursor de $\mathrm{LaFe}_{0,2} \mathrm{Co}_{0,8} \mathrm{O}_{3}$.

\section{Resultados y discusión}

El diagrama FT-IR de la Figura 1, muestra la presencia de señales vibracionales asociadas a grupos funcionales tipo citrato. La señal presente a 3430 $\mathrm{cm}^{-1}$ se puede asociar a modos vibracionales de tensión del enlace $O-H$ de grupos hidroxilos. A 2892 
$\mathrm{cm}^{-1}$ se observa una banda asociada al modo de tensión del enlace $O-H$ de ácidos carboxílicos [18-19]. Las bandas ubicadas hacia 2344 y $2358 \mathrm{~cm}^{-1}$, corresponden con modos de tensión asociados a la presencia de $\mathrm{CO}_{2}$, Así mismo, la señal ubicada a 1585 $\mathrm{cm}^{-1}$, puede asociarse al modo de tensión $C=O$. Las bandas a 1328 y $1400 \mathrm{~cm}^{-1}$, pueden corresponder con el modo de tensión simétrico del $\mathrm{COO}^{-}$. Las señales localizadas a 1257,1108 y $929 \mathrm{~cm}^{-1}$, corresponden al modo de tensión del enlace $C$ - $O$, que generalmente se forma en el secado de los soles de ácido cítrico [20-22]. Las bandas ubicadas a 829, 713 , y $492 \mathrm{~cm}^{-1}$, corresponden a los enlaces $\mathrm{M}-\mathrm{OH}$ y $M-O$ de los diferentes cationes metálicos de $L a^{+3}$, $\mathrm{Fe}^{+3}$, y $\mathrm{Co}^{+3}[23,24]$.

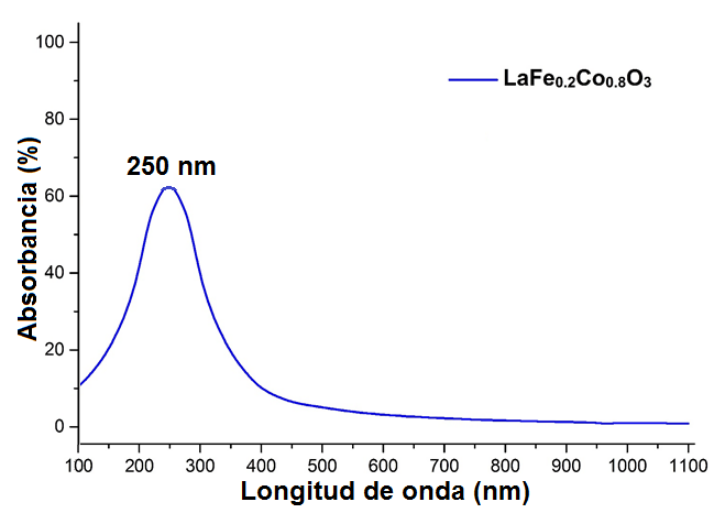

Figura 2. Espectro UV-vis del precursor de $\mathrm{LaFe}_{0,2} \mathrm{Co}_{0,8} \mathrm{O}_{3}$.

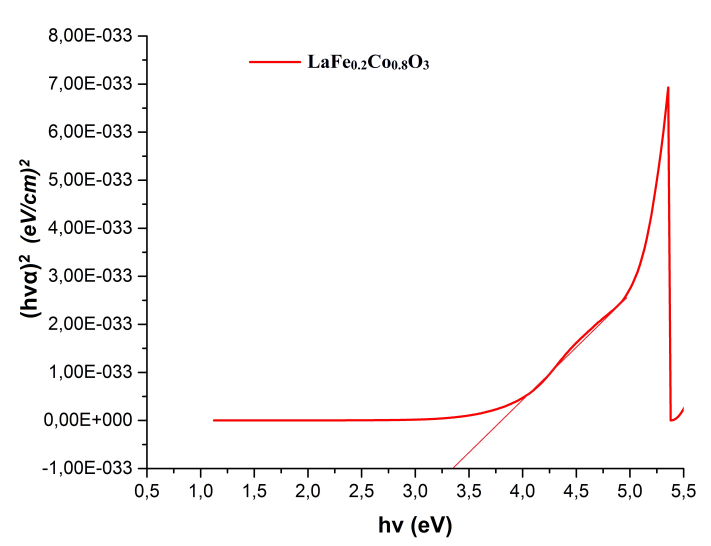

Figura 3. Diagrama $(h v \alpha)^{2}$ vs $h v$ del precursor de $\mathrm{LaFe}_{0,2} \mathrm{Co}_{0,8} \mathrm{O}_{3}$

El análisis por espectroscopía UV-vis del precursor metalorgánico de la Figura 2, confirma que las principales transiciones electrónicas se alcanzan alrededor de los $231 \mathrm{~nm}$, lo que indica que son altamente energéticas y que se relacionan con las transiciones electrónicas entre el oxoanión de citrato y los diferentes cationes metálicos alrededor de $\lambda=200 \mathrm{~nm}$. En base a esta banda de absorción principal, se puede señalar que las especies derivadas de citrato tienden a estabilizarse en forma de aconitatos por la fuerte deshidratación sufrida por el precursor. Lo anterior aporta evidencia acerca de la evolución del precursor de citrato por efecto térmico, conformando un compuesto de enlaces dobles insaturados responsables del movimiento de la banda de transición típica para citratos localizada a $250 \mathrm{~nm}$. El análisis de la Figura 3, realizada a partir de los datos de la gráfica de la Figura 2, arroja una energía de band gap de 3,3 eV , lo que define al cerámico como un óxido semiconductor [15].

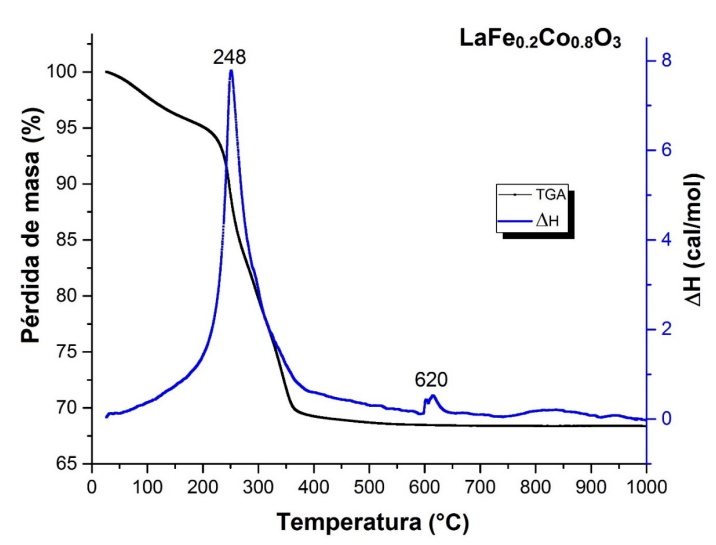

Figura 4. Diagramas TGA-DTA del precursor de $\mathrm{LaFe}_{0,2} \mathrm{Co}_{0,8} \mathrm{O}_{3}$.

El análisis térmico gravimétrico y el térmico diferencial de la Figura 4, muestran que existe una pérdida inicial de masa entre los 50 y $150{ }^{\circ} \mathrm{C}$, asociada con la eliminación de humedad y agua intermolecular [25]. A partir de los $170{ }^{\circ} \mathrm{C}$, se inicia una marcada pérdida de masa que alcanza cerca de un $33 \%$, relacionada con el inicio de procesos de autocombustión y de transformación de los compuestos de coordinación metal-citrato a aconitato, finalizando hacia los $330^{\circ} \mathrm{C}$. Los productos formados al final de esta etapa, principalmente carbonatos y oxo-carbonatos, permanecen estables hasta valores de temperatura de $700^{\circ} \mathrm{C}$, al final de la cual la evidencia de estas especies desaparece y permite la consolidación de las fases cristalinas. Esta temperatura permite establecer las condiciones básicas de síntesis de este material para evitar la sobre-densificación del sólido y la pérdida de propiedades de superficiales [26]. Se observa también una señal exotérmica cercana a los 
$248^{\circ} \mathrm{C}$ relacionada con transformaciones de los compuestos de coordinación metal-citrato, y otro pico exotérmico a $620^{\circ} \mathrm{C}$ asociado con transformaciones de carbonatos y oxo-carbonatos [27].

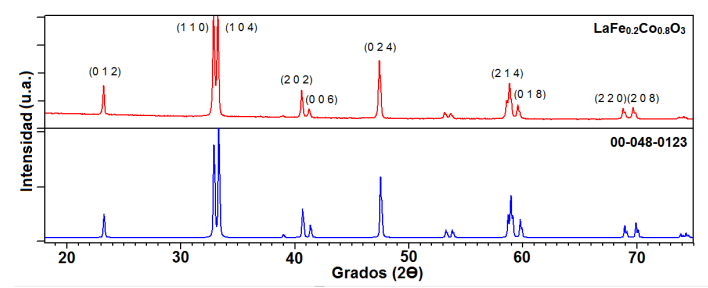

Figura 5. Patrón de difracción de rayos $\mathrm{X}$ del sistema $\mathrm{LaFe}_{0,2} \mathrm{Co}_{0,8} \mathrm{O}_{3}$.

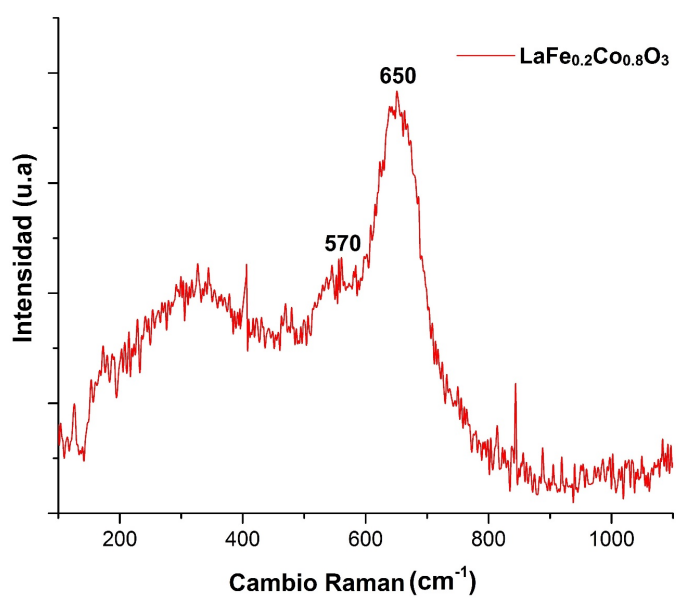

Figura 6. Espectro Raman de $\mathrm{LaFe}_{0,2} \mathrm{Co}_{0,8} \mathrm{O}_{3}$

El análisis estructural, desarrollado mediante difracción de rayos $\mathrm{X}$ en la muestra calcinada en aire a $850^{\circ} \mathrm{C}$ durante 12 horas, y mostrado en la Figura 5, demuestra la obtención de una única fase predominante, y que concuerdan con el espectro de la estructura de referencia $\mathrm{LaCoO}_{3}(J C P D S=00-048-0123)$ que presenta como parámetros cristalográficos: sistema cristalino romboédrico, grupo espacial $R$-3c (167), $a=5.4445 \AA, b=5.4445 \AA, c=13.0936$ $\AA$ y volumen de celda $=336 \AA^{3}$, con orientación cristalográfica principal hacia los planos $\left(\begin{array}{lll}1 & 1 & 0\end{array}\right)$ y (ll $\left.\begin{array}{ll}1 & 0\end{array}\right)$, acorde con reportes preliminares relacionados con la obtención de este tipo de composiciones, evidenciando la efectividad del método en la síntesis de una composición de una fase pura [28-30]. La determinación del tamaño de cristal, utilizando la ecuación de Debye-Scherrer y una constante de 0,89 como referencia, permitió establecer un tamaño de $31 \mathrm{~nm}[31]$.
Los espectros Raman de la Figura 6, confirman la formación del óxido $\mathrm{LaCoO}_{3}$, gracias a la presencia de dos señales intensas localizada a 570 y $650 \mathrm{~cm}^{-1}$ que concuerdan con modos vibracionales de tensión $E_{g}$ para materiales con contenidos de lantano y cobalto en los sitios catiónicos $A$ y $B$ respectivamente, dentro de la estructura tipo $A B O_{3}$ romboédrica de espacio grupal $R-3 c$, cuyos modos activos para esta técnica son $A_{1 g}+4 E_{g}[28,32-35]$.

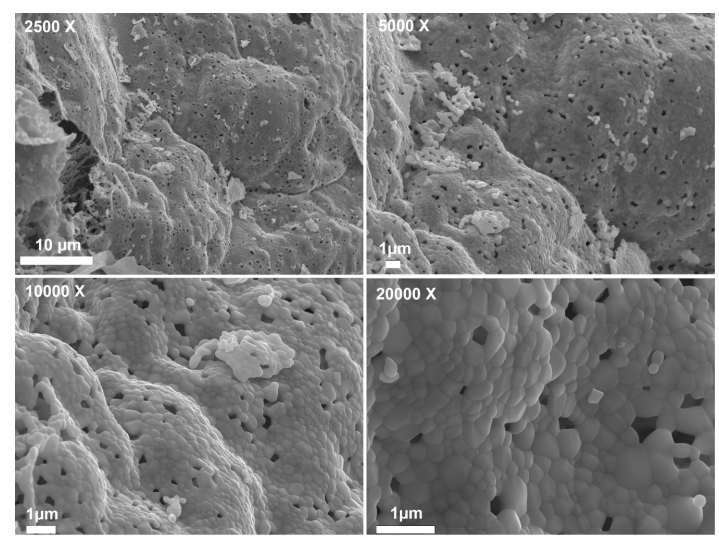

Figura 7. Imágenes de $\mathrm{LaFe}_{0,2} \mathrm{Co}_{0,8} \mathrm{O}_{3}$ a diferentes aumentos.

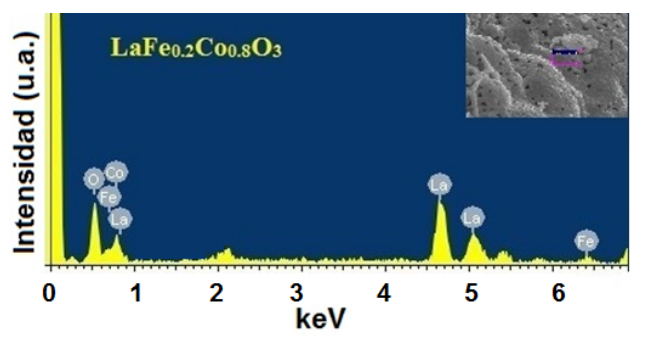

Figura 8. Espectro de energía dispersiva de Rayos X de $\mathrm{LaFe}_{0,2} \mathrm{Co}_{0,8} \mathrm{O}_{3}$

La Figura 7 muestra las imágenes obtenidas a través de microscopia electrónica de barrido de la superficie de la muestra del material a diferentes magnificaciones. Como allí se observa, la ruta de síntesis empleada permitió la formación de materiales con morfología y distribución de partícula homogéneas y claramente distinguibles, con tamaño menores a $1 \mu \mathrm{m}$, y alto grado de porosidad, originadas por la descomposición de compuestos volátiles en los pasos de autocombustión y calcinación de los polvos cerámicos, y que favorecen la difusión de combustibles gaseosos dentro de procesos de oxidación [36]. Para generar un perfil composicional del cerámico, se aplicó la técnica de energía dispersiva de rayos X, dando como resultado emisiones de los elementos 
lantano, cobalto, hierro y oxígeno. Como se ve en la Figura 8, no se encontraron elementos ajenos a la estructura buscada, lo que corrobora la efectividad el método de polimerización con ácido cítrico en el logro de composiciones con fases puras y uniformes dentro del material [37-38].

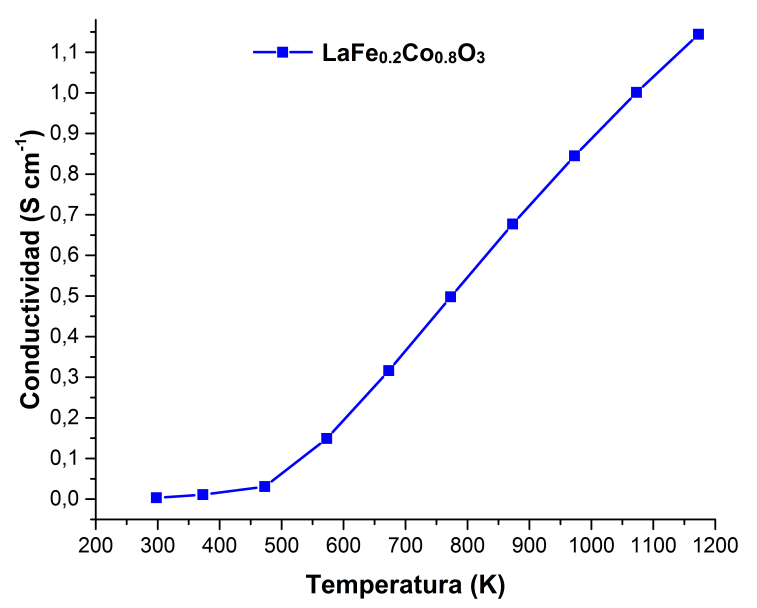

Figura 9. Gráfica de conductividad desde $25 \check{\mathrm{r} C}$ hasta $900 \check{\mathrm{C} C}$ de $\mathrm{LaFe}_{0,2} \mathrm{Co}_{0,8} \mathrm{O}_{3}$.

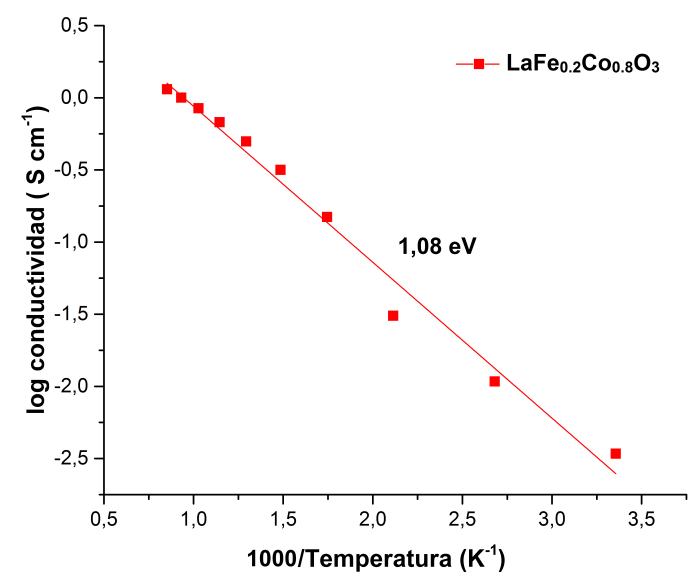

Figura 10. Gráfica tipo Arrhenius de $\mathrm{LaFe}_{0,2} \mathrm{Co}_{0,8} \mathrm{O}_{3}$.

A partir de los datos de la Figura 9, donde se muestran que a $25^{\circ} \mathrm{C}$ se alcanzó una conductividad de 3,41 x $10^{-3} \mathrm{~S} \mathrm{~cm}^{-1}$, y a $900^{\circ} \mathrm{C}$ otra de $1,144 \mathrm{~S} \mathrm{~cm}^{-1}$, se encuentra una estrecha relación entre el incremento de la conductividad y el aumento de la temperatura. La gráfica tipo Arrhenius de la Figura 10 presenta una tendencia lineal, cuya pendiente permite determinar un valor de energía de activación de $1,08 \mathrm{eV}$, lo que indica que la conductividad se genera en mayor medida mediante mecanismos electrónicos. El comportamiento mostrado por la Figura 9 y la Figura 10, sumado a los datos obtenidos a partir de la
Figura 3, corroboran la naturaleza eléctrica del material, que se explica gracias a la activación térmica que propicia una mayor movilidad de los portadores de carga a través de los enlaces B-O-B, ayudando a confirmar la estabilidad química y estructural a altas temperaturas [31, 39]. Estos fenómenos son típicos de óxidos semiconductores tipo-p, dentro de los que podemos nombrar a los sistemas $\mathrm{LaMn}_{1-x} \mathrm{Cr}_{x} \mathrm{O}_{3 \pm \delta}$, $\mathrm{LaFe}_{1-x} \mathrm{Co}_{x} \mathrm{O}_{3 \pm \delta}$, y $\mathrm{LaFe}_{1-x} \mathrm{Ti}_{x} \mathrm{O}_{3 \pm \delta}$, que han sido ampliamente estudiados por sus interesantes propiedades eléctricas, derivadas del uso de rutas de síntesis de química húmeda [40-43].

\section{Conclusiones}

Los análisis con espectroscopia infrarroja, térmicos gravimétricos y diferenciales, y espectroscopia ultravioleta visible del precursor tipo citrato del cerámico $\mathrm{LaFe}_{0,2} \mathrm{Co}_{0,8} \mathrm{O}_{3}$, comprobaron la efectividad del método de síntesis de polimerización con ácido cítrico para alcanzar la completa disolución de los cationes de lantano, hierro y cobalto dentro de la estructura tipo perovskita, al igual que un gran control sobre la composición buscada y el establecimiento de una temperatura de formación cristalina estable del material a partir de $700^{\circ} \mathrm{C}$. Los análisis de difracción de rayos $\mathrm{X}$, espectroscopia Raman y microscopia electrónica de barrido, mostraron la formación de una fase única, junto con una alta homogeneidad en la forma, tamaño y distribución de las partículas. Mediante la ecuación de Debye-Scherrer se calculó un tamaño de cristal de $31 \mathrm{~nm}$, logrando una gran área superficial, activa para procesos catalíticos de oxidación de combustibles, además de una porosidad que facilita el transporte de iones y electrones. Se encontró también, aplicando espectroscopia de impedancias, que la conductividad electrónica se incrementa con el aumento de la temperatura, alcanzando un valor de $1,144 \mathrm{~S} \mathrm{~cm}^{-1}$ a $900^{\circ} \mathrm{C}$ y una energía de activación de 1,08 eV; confiriéndole al óxido un comportamiento típico de semiconductores tipo- $p$, y alta estabilidad química y estructural en un amplio rango de temperaturas.

\section{Referencias}

[1] Steven McIntosh, Raymond J. Gorte, "Direct Hydrocarbon Solid Oxide Fuel Cells", Chem. Rev., vol. 104, pp. 4845-4865, 2004. Disponible en: http://dx.doi.org/10.1021/cr020725g 
[2] Nam-Gyu Park, "Perovskite solar cells: an emerging photovoltaic technology". Materials Today, vol. 18 , no. 2, pp. 6572, 2015. Disponible en: http://dx.doi.org/ 10.1016/j.mattod.2014.07.007

[3] Fengyu Shen, Kathy Lu, "Comparative study of $\mathrm{La}_{0.6} \mathrm{Sr}_{0.4} \mathrm{Co}_{0.2} \mathrm{Fe}_{0.8} \mathrm{O}_{3}, \mathrm{Ba}_{0.5} \mathrm{Sr}_{0.5} \mathrm{Co}_{0.2}$ $\mathrm{Fe}_{0.8} \mathrm{O}_{3}$ and $\mathrm{Sm}_{0.5} \mathrm{Sr}_{0.5} \mathrm{Co}_{0.2} \mathrm{Fe}_{0.8} \mathrm{O}_{3}$ cathodes and the effect of $\mathrm{Sm}_{0.2} \mathrm{Ce}_{0.8} \mathrm{O}_{2}$ block layer in solid oxide fuel cells", International journal of hydrogen energy, vol. 40, pp. 1645716465, 2015. Disponible en: http://dx.doi. org/10.1016/j.ijhydene.2015.09.148

[4] Carlos Moure; Octavio Peña., "Recent advances in perovskites: Processing and properties", Progress in Solid State Chemistry, vol. 43 , no. 2 , pp. 123-148, 2015. Disponible en: http://dx.doi.org/10.1016/j.progsolidstchem.201 5.09 .001

[5] Christopher J. Benedict, Ashok Rao, Ganesh Sanjeev, G. S. Okram, P. D. Babu, "A systematic study on the effect of electron beam irradiation on structural, electrical, thermo-electric power and magnetic property of $\mathrm{LaCoO}_{3}$ ", Journal of Magnetism and Magnetic Materials, vol. 397, pp. 145-151, 2016. Disponible en: http://dx.doi.org/10.1016/j.jmmm.2015.08.111

[6] V. Mehta, S. Bose, J. M. Iwata-Harms, E. Arenholz, C. Leighton, Y. Suzuki, Phys. Rev. B, vol. 87, 2013.

[7] L. Predoana, B. Malic, D. Crisan, N. Dragan, M. Anastasescu, J. CalderonMoreno, R. Scurtu, M. Zaharescu, " $\mathrm{LaCoO}_{3}$ ceramics obtained from reactive powders", Ceramics International, vol. 38, pp. 5433-5443, 2012. Disponible en: http://dx.doi.org/10.1016/j.ceramint.2012.03.054

[8] Ahmed Galal, Nada F. Atta, Shimaa M. Ali, "Investigation of the catalytic activity of $\mathrm{LaBO}_{3}$ $(\mathrm{B}=\mathrm{Ni}, \mathrm{Co}, \mathrm{Fe}$ or $\mathrm{Mn})$ prepared by the microwave-assisted method for hydrogen evolution in acidic medium", Electrochimica Acta, vol. 56, pp. 5722-5730, 2011. Disponible en: http://dx.doi.org/10.1016/j.electacta.2011.04.045

[9] J. Alvarado-Flores, L. Ávalos-Rodríguez, "Materiales para ánodos, cátodos y electrolitos utilizados en celdas de combustible de óxido sólido
(SOFC)", Revista Mexicana de Física, vol. 59, pp. 66-87, 2013.

[10] H. C. Chang, Solid State Ionics, vol. 180, pp. 412-417, 2009.

[11] S. Gaikward, S. Dhesphande, Y. Khollam, S. Violet, V. Ravi, "Coprecipitation method for the preparation ofnanocrystalline ferroelectric $\mathrm{CaBi}_{2} \mathrm{Ta}_{2} \mathrm{O}_{9}$ ", Mater. Lett., vol. 58, no. 27-28, pp. 3474-3476, 2004. Disponible en: http://dx.doi.org/10.1016/j.matlet.2004.07.004.

[12] L. Predoana, B. Malic, D. Crisan, N. Dragan, M. Anastasescu, J. Calderon-Moreno, R. Scurtu, M. Zaharescu, " $\mathrm{HaCoO}_{3}$ ceramics obtained from reactive powders", $\mathrm{Ce}$ ramics International, vol. 38, pp. 54335443, 2012. Disponible en: http://dx.doi.org/ 10.1016/j.ceramint.2012.03.054

[13] José Juan Alvarado Flores, Ilya Espitia Cabrera, Jaime Espino Valencia y Armando Reyes Rojas, "Impregnación de la perovskita $\mathrm{La}_{0.8} \mathrm{Sr}_{0.2} \mathrm{Cr}_{0.5} \mathrm{Mn}_{0.5} \mathrm{O}_{3-\delta}$ como ánodo en celdas SOFC", Boletín de la sociedad española de cerámica y vidrio, vol. 54, pp. 198-208, 2015. Disponible en: http://dx.doi.org/10.1016/j.bsecv.2015.08.001

[14] Z. Shao, W. Zhou, Z. Zhu, "Advanced synthesis of materialsfor intermediate-temperature solid oxide fuel cells", Prog. Mater. Sci., vol. 57, no. 4, pp. 804-874, 2012. Disponible en: http://dx.doi.org/10.1016/j.pmatsci.2011.08.002.

[15] E. García-López, G. Marcì, F. Puleo, V. La Parola, L. F. Liotta, " $L a_{1 ? x} S r_{x} \mathrm{Co}_{1 ? y} \mathrm{Fe}_{y} \mathrm{O}_{3-\delta}$ perovskites: Preparation, characterization and solar photocatalytic activity", $A p$ plied Catalysis B: Environmental, vol. 178, pp. 218-225, 2015. Disponible en: http://dx.doi.org/10.1016/j.apcatb.2014.09.014

[16] Xiaokun Yang, Lisha Yang, Wei Fan, Hongfei Lin., "Effect of redox properties of $\mathrm{aCoO}_{3}$ perovskite catalyst on production of lactic acid from cellulosic biomass", Catalysis Today, vol. 269, pp. 56-64, 2016. Disponible en: http://dx.doi.org/10.1016/j.cattod.2015.12.003

[17] Daniel D. Athayde, Douglas F. Souza, Alysson M. A. Silva, Daniela Vasconcelos, Eduardo H. M. Nunes, João C. Diniz da Costa, Wander L. Vasconcelos. "Review of perovskite ceramic synthesis and membrane 
preparation methods", Ceramics International, vol. 42, pp. 6555-6571, 2016. Disponible en: http://dx.doi.org/10.1016/j.ceramint.2016.01.130

[18] Elham Ghiasi, Azim Malekzadeh, Mahnaz Ghiasi, "Moderate concentration of citric acid for the formation of $\mathrm{LaMnO}_{3}$ and $\mathrm{LaCoO}_{3}$ nano-perovskites", Journal of Rare Earths, vol. 31, no. 10, pp. 997-1002, 2013. Disponible en: http://dx.doi.org/10.1016/S10020721(12)60393-7

[19] Refka Andoulsi, Karima Horchani-Naifer, Mokhtar Férid, "Effect of the preparation route on the structure and microstructure of $\mathrm{LaCoO}_{3}$ ". Chemical Papers, vol. 68, no. 5, pp. 608-613, 2014. Disponible en: http://dx.doi.org/10.2478/s11696-013-0490-x

[20] A.Worayingyong, P. Kangvansura, S. Kityakarn, Schiffbase, "Complex sol-gel method for $\mathrm{LaCoO}_{3}$ perovskite preparation with highadsorbed oxygen colloids", Surf. A: Physicochem. Eng. Asp., vol. 320, pp. 123-129, 2008.

[21] K. S Weil, J. S. Hardy, J. Y. Kim, "A new technique for joining ceramic and metal components in high temperature electrochemical devices", J. Adv. Mater., vol. 2, pp. 84-94, 2007.

[22] M. T. Tsai, "Effects of hydrolysis processing on the character of forsterite gel fibers. Part I: preparation, spinnability and molecular structure", Journal of the European Ceramic Society, vol. 22, pp. 1073-1083. 2002. Disponible en: http://dx.doi.org/10.1016/s09552219(01)00417-4.

[23] F. Kousar, S. Nazim, M. F. Warsi, M. A. Khan, M. N. Ashiq, Z. A. Gilani,I. Shakir, A.Wadood, " $\mathrm{La}_{1-x} \mathrm{E} u_{x} \mathrm{FeOs} s_{3}$ nanoparticles: Fabrication via micro-emulsion route for high frequency devices applications", J.Alloy.Compd., vol. 629, pp. 315-318, 2015. Disponible en: http://refhub.elsevier.com/S03048853(15)30167-0/sbref11

[24] N. N. Lubinskii,, L. A. Bashkirov , G. S. Petrov , S. V. Shevchenko, I. N. Kandidatova, M. V. Bunshinskii, "Crystal structure and IR spectra of lanthanum cobaltites-gallates", Glass and Ceramics, vol. 66, pp. 59-62, 2009. Disponible en: http://dx.doi.org/10.1007/s10717-0099124-8.
[25] R. Brackmann, C. A. Perez, M. Schmal., " $\mathrm{LaCoO}_{3}$ perovskite on ceramic monoliths - Pre and post reaction analyzes of the partial oxidation of methane". International journal of hydrogen energy, vol. 39, pp. 13991-14007, 2014. Disponible en: http://dx.doi.org/10.1016/j.ijhydene.2014.07.027

[26] Qurshia Choudhry, Muhammad Azhar Khan, Gulfam Nasar, Azhar Mahmood, Muhammad Shahid, Imran Shakir, Muhammad Farooq Warsi, "Synthesis, characterization and study of magnetic, electrical and dielectric properties of $\mathrm{La}_{1-x} \mathrm{Dy}_{x} \mathrm{Co}_{1-y} \mathrm{Fe}_{y} \mathrm{O}_{3}$ nanoparticles prepared by wet chemical route". Journal of Magnetism and Magnetic Materials, vol. 393, pp. 67-72, 2015. Disponible en: http://dx.doi.org/10.1016/j.jmmm.2015.05.040

[27] A. Mahmood, M. Nadeem, B. Bashir, I. Shakir, M. N. Ashiq, M. Ishaq, A. Jabbar, R. Parveen, M. Shahid, M. F. Warsi, "Synthesis,characterization and studies of various structural, physical, magnetic, electrical and dielectric parameters for $L a_{1-x} D y_{x} N i_{1-y} M n_{y} O_{3}$ nanoparticles", J. Magn. Magn. Mater. vol. 348, pp. 82-87, 2013. Disponible en: $\quad$ http://refhub.elsevier.com/S03048853(15)30167-0/sbref14

[28] Settakorn Upasen, Pierre Batocchi, Fabrice Mauvy, Aneta Slodczyk, Philippe Colomban, "Chemical andstructuralstabilityof $\mathrm{La}_{0.6} \mathrm{Sr}_{0.4} \mathrm{Co}_{0.2} \mathrm{Fe}_{0.8} \mathrm{O}_{3-\delta}$ ceramic vs. medium/high water vapor pressure". Ceramics International, vol. 41, pp. 1413714147, 2015. Disponible en: http://dx.doi.org/ 10.1016/j.ceramint.2015.07.035

[29] B. Philippeau, F. Mauvy, C. Mazataud, S. Fourcade, J. C. Grenier, "Comparative study of electrochemical properties of mixed conducting $\mathrm{Ln}_{2} \mathrm{NiO}_{4+\delta}(\mathrm{Ln}=\mathrm{La}$, $\mathrm{Pr}, \quad \mathrm{Nd}) \quad$ and $\mathrm{La}_{0.6} \mathrm{Sr}_{0.4} \mathrm{Co}_{0.2} \mathrm{Fe}_{0.8} \mathrm{O}_{3+\delta}$ as SOFC cathodes associated to $\mathrm{Ce}_{0.9} \mathrm{Gd}_{0.1} \mathrm{O}_{2-\delta}, \quad \mathrm{La}_{0.8} \mathrm{Sr} 0.2 \mathrm{Ga}_{0.8} \mathrm{Mg}_{0.2} \mathrm{O}_{3-\delta}$ and $\mathrm{La}_{9} \mathrm{Sr}_{1} \mathrm{Si}_{6} \mathrm{O}_{26.5}$ electrolytes", Solid State Ion., vol. 249-250, pp. 17-25, 2015. Disponible en: http://refhub.elsevier.com/S02728842(15)01321-8/sbref3

[30] C. C. Wang, T. Becker, K. Chen, L. Zhao, B. Wei, S. P. Jiang, "Effect of temperatura on the chromium deposition and poi- 
soning of $\mathrm{La}_{0.6} \mathrm{Sr}_{0.4} \mathrm{Co}_{0.2} \mathrm{Fe}_{0.8} \mathrm{O}_{3-\delta}$ cathodes of solid oxide fuel cells", Electrochim. Acta, vol. 139, pp. 173-179, 2014. Disponible en: http://refhub.elsevier.com/S02728842(15)01321-8/sbref35

[31] Augusto Mejía Gómeza, Joaquín Sacanella, Ana Gabriela Leyva, Diego G. Lamas, "Performance of $\mathrm{La}_{0.6} \mathrm{Sr}_{0.4} \mathrm{Co}_{1-y} \mathrm{Fe}_{y} \mathrm{O}_{3}$ (y = $0.2,0.5$ and 0.8 ) nanostructured cathodes for intermediate-temperature solid-oxide fuel cells: Influence of microstructure and composition", Ceramics International, vol. 42, pp. 3145-3153, 2016. Disponible en: http://dx.doi.org/10.1016/j.ceramint.2015.10.104

[32] E. Siebert, A. Boréave, F. Gaillard, T. Pagnier, "Electrochemical and Raman study of $\mathrm{La}_{0.7} \mathrm{Sr}_{0.3} \mathrm{Co}_{0.8} \mathrm{Fe}_{0.2} \mathrm{O}_{3-\delta} \quad$ reduction", Solid State Ion., vol. 247248, pp. 30-40, 2013. Disponible en: http://dx.doi.org/10.1016/j.ssi.2013.05.006

[33] W. Araki, T. Yamaguchi, Y. Arai, J. Malzbender, "Strontium Surface segregation in $\mathrm{La}_{0.58} \mathrm{Sr}_{0.42} \mathrm{Co}_{0.2} \mathrm{Fe}_{0.8} \mathrm{O}_{3-\delta} \quad$ annealed under compression", Solid State Ion., vol. 268, Part A, pp.1-6, 2014. Disponible en: http://refhub.elsevier.com/S02728842(15)01321-8/sbref38

[34] Md. Motin Seikh, L. Sudheendra, Chandrabhas Narayana, C.N.R. Rao, "A Raman study of the temperature-induced lowto-intermediate-spin state transition in $\mathrm{LaCoO}_{3}$ ", Journal of Molecular Structure, vol. 706, pp. 121-126, 2004. Disponible en: http://dx.doi.org/10.1016/j.molstruc.2004.03.058

[35] Nguyen Van Minh, In-Sang Yang, "A Raman scattering study of structural changes in $\mathrm{LaMn}_{1-x} \mathrm{Co}_{x} \mathrm{O}_{3+\delta}$ system", Vibrational Spectroscopy, vol. 42, pp. 353-356, 2006. Disponible en: http://dx.doi.org/10.1016/j.vibspec.2006.05.027

[36] Fabio Souza Toniolo, Robert Newton S.H. Magalhaes, Carlos Andre C. Perez, Martin Schmal, "Structural investigation of $\mathrm{LaCoO}_{3}$ and $\mathrm{LaCoCuO}_{3}$ perovskite-type oxides and the effect of $\mathrm{Cu}$ on coke deposition in the partial oxidation of methane". Applied Catalysis B: Environmental, vol. 117-118, pp. 156-166, 2012. Disponible en: http://dx.doi.org/10.1016/j.apcatb.2012.01.009
[37] A. F. Cruz Pacheco, J. A. Gómez Cuaspud, "Síntesis y caracterización del sistema $\mathrm{La}_{0,8} \mathrm{Sr}_{0,2} \mathrm{MnO}_{3}$ ", Ciencia en Desarrollo, vol. 6, no. 2, pp. 133-139, 2015. Disponible en: http://dx.doi.org/10.19053/01217488.3783

[38] O. C. Vergara Estupiñan, J. A. Gómez Cuaspud, "Síntesis y caracterización de la Perovskita $\mathrm{La}_{0,95} \mathrm{Sr}_{0,05} \mathrm{CrO}_{3}$ ”, Ciencia en Desarrollo, vol. 6, no. 1, pp. 119-125, 2015. Disponible en: http://dx.doi.org/10.19053/01217488.3656

[39] A. Reyes-Rojas, J. Alvarado-Flores, H. Esparza-Poncea, M. Esneider-Alcala, I. Espitia-Cabrera, E. Torres-Moye. "Symmetry breaking and electrical conductivity of $\mathrm{La}_{0.7} \mathrm{Sr}_{0.3} \mathrm{Cr}_{0.4} \mathrm{Mn}_{0.6} \mathrm{O}_{3-\delta}$ perovskite as SOFC anode material", Materials Chemistry and Physics, vol. 126, pp. 773-779, 2011. Disponible en: http://dx.doi.org/10.1016/j.matchemphys.2010.1 2.045

[40] Junling Meng, Xiaojuan Liua, Chuangang Yao, Xiong Zhang, Xiliang Liu, Fanzhi Meng, Jian Meng, "Investigations on structures, thermal expansion and electrochemical properties of $\mathrm{La}_{0.75} \mathrm{Sr}_{0.25} \mathrm{Cu}_{0.5-x} \mathrm{Co}_{x} \mathrm{Mn}_{0.5} \mathrm{O}_{3-\delta}$ $(\mathrm{x}=0,0.25$, and 0.5$)$ as potential cathodes for intermediate temperature solid oxide fuel cells", Electrochimica Acta, vol. 186, pp. 262-270, 2015. Disponible en: http://dx.doi.org/10.1016/j.electacta.2015.10.166

[41] Byung Hyun Park, Gyeong Man Choi., "Ex-solution of $\mathrm{Ni}$ nanoparticles in a $\mathrm{La}_{0.2} \mathrm{Sr}_{0.8} \mathrm{Ti}_{1-x} \mathrm{Ni}_{x} \mathrm{O}_{3-\delta}$ alternative anode for solid oxide fuel cell", Solid State Ionics, vol. 262, pp. 345-348, 2014. Disponible en: http://dx.doi.org/10.1016/j.ssi.2013.10.016

[42] Phokha Sumalin, Hunpratup Sitchai, Pinitsoontorn Supree, Putasaeng Bundit, Rujirawat Saroj, Maensiri Santi, "Structure, magnetic, and dielectric properties of Ti-doped $\mathrm{LaFeO}_{3}$ ceramics synthesized by polymer pyrolysis method", Materials Research Bulletin, vol. 67, pp. 118-125, 2015. Disponible en: 10.1016/j.materresbull.2015.03.008

[43] Devinder Singh, Arun Mahajan, "Effect of A-site cation size on the structural, magnetic, and electrical properties of $\quad \mathrm{La}_{1-x} \mathrm{Nd}_{x} \mathrm{Mn}_{0.5} \mathrm{Cr}_{0.5} \mathrm{O}_{3}$ perovskites", Journal of Alloys and Compounds, vol. 
Wilson Rativa Parada, et al.

644, pp. 172-179, 2015. Disponible en:

https://doi.org/10.1016/j.jallcom.2015.04.180 